\title{
Clinicopathologic characteristics and prognostic factors for HER2-positive patients with metastatic breast cancer in southern China
}

\author{
Tao Qin' ${ }^{1}$, Zhong-Yu Yuann ${ }^{1}$, Rou-Jun Peng ${ }^{1}$, Bing Bai ${ }^{1}$, Yin-Duo Zeng ${ }^{2}$, Yan-Xia Shi ${ }^{1}$, Xiao-Yu Teng ${ }^{1}$, \\ Dong-Geng Liu', Shu-Sen Wang ${ }^{1}$
}

\author{
1Department of Medical Oncology, State Key Laboratory of Oncology in South China, \\ Sun Yat-sen University Cancer Center, Guangzhou, China \\ ${ }^{2}$ Breast Tumor Center, Sun Yat-sen Memorial Hospital, Sun Yat-sen University, \\ Guangzhou, China
}

Submitted: 25 January 2013

Accepted: 8 June 2013

Arch Med Sci 2015; 11, 3: 544-550

DOI: $10.5114 /$ aoms.2015.52356

Copyright @ 2015 Termedia \& Banach

\section{Abstract}

Introduction: The aim of the study was to analyze clinicopathologic characteristics and survival and to identify prognostic factors for Chinese patients with HER2-positive metastatic breast cancer.

Material and methods: A total of 243 patients with HER2-positive metastatic breast cancer, treated during the period 2002 to 2009, were followed up from initial disease diagnosis to death or date of last follow-up (December 2011). Cumulative survival curves were created using Kaplan-Meier analysis with the log-rank test. Prognostic factors were analyzed by univariate and multivariate Cox proportional hazards regression analysis.

Results: During follow-up, 205 patients died, with a median OS of 27 months (95\% Cl: $23.5,30.5$ months), and the 1-, 3-, and 5-year survival rates were $84.4 \%, 38.6 \%$, and $18.1 \%$, respectively. The median OS of HR+ patients was significantly higher than that of HR- patients $(p<0.001)$. Surgery (hazard ratio $=0.60, p=0.002$ ), endocrine therapy (hazard ratio $=0.53, p<0.01$ ), and anti-HER2 therapy (hazard ratio $=0.63, p=0.003$ ) were favorable independent prognostic factors for patients with HER2-positive metastatic breast cancer.

Conclusions: These results indicated that surgical intervention, endocrine therapy, and anti-HER2 therapy were good for these HER2 positive patients with metastatic breast cancer, but ECOG performance status $<1$ and metastasis to brain were unfavorable independent prognostic factors. HR status was not an independent prognostic factor.

Key words: anti-HER2 therapy, brain metastasis, endocrine therapy, hormone receptor status, surgery.

\section{Introduction}

Breast cancer is the most common cancer among women worldwide, with the greatest incidences in 2008 occurring in more developed regions (e.g., 89.1 per 100,000, age adjusted, in the United Kingdom and 76 per 100,000 in the United States) [1]. The incidence of breast cancer among women is lower in Asian countries, with an estimated incidence in China in 2008 of 21.6 per 100,000 [1]; however, the incidence in less developed regions is increasing. Mortality is similar among countries,

\author{
Corresponding author: \\ Shu-Sen Wang MD \\ Department \\ of Medical Oncology \\ State Key Laboratory \\ of Oncology in South China \\ Sun Yat-Sen University \\ Cancer Center \\ No. 651 East Dongfeng Road \\ Guangzhou, China \\ Phone: +86-020-87343794 \\ Fax: +86-020-87342694 \\ E-mail: \\ wangshs@sysucc.org.cn
}


likely due to greater access to newer therapies in more developed countries [1].

In addition to prognostic factors such as age, tumor size, and cancer stage and grade, overexpression of the human epidermal growth factor receptor 2 oncogene (HER2-positive [HER2+]) and positivity for estrogen receptor (ER) or progesterone receptor (PR) (referred to as hormone receptor-positive $[\mathrm{HR+}]$ ) are heavily studied prognostic factors. HER2 overexpression occurs in up to $30 \%$ of breast cancers and is associated with poor prognosis, predicting decreased overall survival (OS) and time to relapse [2, 3]. However, the introduction of trastuzumab therapy has drastically altered this situation, significantly increasing outcomes in HER2+ patients [4], although significant cardiac complications can occur [5]. HR status is also prognostic, with $\mathrm{HR}$ - patients having a poorer prognosis compared to $\mathrm{HR}+$ patients [6]. Recent studies indicate that this relation extends to the population of HER2+ patients [7-11].

Studies identifying prognostic factors for Chinese patients with various types of breast cancer have been published [12,13], and some have identified specifically HER2 overexpression and younger age at diagnosis as associated with poorer survival [14-19]. However, few, if any, have evaluated prognostic factors specifically for Chinese patients with HER2+ metastatic breast cancer. Identification of prognostic clinicopathologic factors among different patient populations could aid in the development of individualized treatment regimens.

The aim of this study was to assess the effects of HR status, surgical intervention, and anti-HER2 therapy on OS of patients with HER2+ metastatic breast cancer in southern China and to identify prognostic clinicopathologic characteristics in this patient population.

\section{Material and methods}

\section{Clinical information}

This is a retrospective study approved by the Institutional Review Board of Sun Yat-sen University. A total of 243 female patients with HER2 positive metastatic breast cancer, who received treatment at Sun Yat-sen University Cancer Center during the period January 2002 to January 2009, were assessed. The inclusion criteria were as follows: known performance status at first diagnosis of metastatic breast cancer, pathologically confirmed breast cancer, defined TNM stage, known HR status, known adjunctive or palliative therapy, known metastatic sites, known survival status, and complete follow-up information. Patients who did not fulfill the inclusion criteria or without complete clinical data were excluded. The primary cancer was confirmed by pathologic examina- tion. TNM staging was performed according to the AJCC Cancer Staging Manual, $7^{\text {th }}$ edition [20]. HER2 overexpression was assessed in well-fixed, paraffin-embedded tumor specimens containing invasive cancer cells. HER2 overexpression was defined as HER2 immunohistochemical staining of $3+(>30 \%$ of invasive cancer cells showing strong membrane staining) or a HER2 : chromosome 17 ratio of $>2.2$ determined by fluorescence in situ hybridization. ER/PR status was determined by immunohistochemistry, with staining of $>10 \%$ of cells defined as positive HR status.

\section{Treatment}

Of the 243 patients, 26 (10.7\%) received a diagnosis of stage IV breast cancer at first diagnosis. The remaining 217 patients were initially diagnosed with stage I to III breast cancer but later developed metastasis. After the diagnosis of metastasis, 214 patients received systemic chemotherapy as follows: paclitaxel for 154 (63.4\%) patients, anthracycline for 73 (30.0\%), capecitabine for 44 (18.1\%), platinum for 41 (16.9\%), vinorelbine for 30 (12.4\%), and gemcitabine for 24 (9.9\%). In addition, 87 (35.8\%) patients received endocrine therapy, in which tamoxifen, aromatase inhibitors (such as anastrozole, letrozole, exemestane), or goserelin was used. Of 85 patients receiving anti-HER2 treatment, trastuzumab or lapatinib was used. Eight patients were treated with bevacizumab. Among 63 patients receiving surgical intervention, 35 had local and regional operations, 24 had distant metastatic lesion removal, and 4 underwent radiofrequency ablation.

Before therapy, each patient's physician(s) informed them that the best strategy for the treatment of metastatic breast cancer for HER2+ patients was treatment targeting HER2. It should be noted that trastuzumab and lapatinib are not covered by insurance in China, and most patients cannot afford treatment. Thus, HER2-targeted therapy is not as commonly performed in China as might be preferred. The risks and benefits of treatment were explained to the patients and/ or their relatives, and informed consent was obtained before treatment.

\section{Follow-up}

Patients were followed every 2 to 3 months at outpatient clinics, where physical and imaging examinations were performed. The primary endpoint in the present study was overall survival (OS), defined as the interval from the initiation of treatment after metastasis to death or the last follow-up.

Evaluation of efficacy was performed according to the criteria of the Response Evaluation Criteria 
in Solid Tumors (RECIST v. 1.0) [21]. Efficacy was classified as complete remission (CR), partial remission (PR), stable disease (SD), or progression of disease (PD). Of 214 patients with recurrence who received systemic chemotherapy, the efficacy of first-line chemotherapy was as high as $58.0 \%$; CR was achieved for 13 patients (6.1\%), PR for 111 (51.9\%), SD for 65 (30.4\%), and PD for 25 (11.7\%). Performance status evaluation was conducted using the Eastern Cooperative Oncology Group (ECOG) score [22].

Table I. Clinical characteristics of 243 patients with HER2-positive metastatic breast cancer

\begin{tabular}{|c|c|}
\hline Clinical characteristic & Total $(n=243)$ \\
\hline Age at metastasis, mean \pm SD [years] & $46.0 \pm 10.1$ \\
\hline \multicolumn{2}{|l|}{ Histologic type, $n(\%)$ : } \\
\hline Invasive ductal & $222(91.4)$ \\
\hline Invasive lobular & $3(1.2)$ \\
\hline Other* & $18(7.4)$ \\
\hline \multicolumn{2}{|l|}{ HR status, $n(\%)$ : } \\
\hline Positive & $138(56.8)$ \\
\hline Negative & $105(43.2)$ \\
\hline Chemotherapy, $n$ (\%) & $214(88.1)$ \\
\hline Radiotherapy, $n$ (\%) & $88(36.2)$ \\
\hline Adjuvant endocrine therapy, $n$ (\%) & $87(35.8)$ \\
\hline Anti-HER2 therapy, $n(\%)$ & $85(35.0)$ \\
\hline Surgery, $n(\%)$ & $63(25.9)$ \\
\hline \multicolumn{2}{|l|}{ ECOG performance status score, $n(\%)$ : } \\
\hline$\leq 1$ & $224(92.2)$ \\
\hline$>1$ & $19(7.8)$ \\
\hline \multicolumn{2}{|l|}{ Metastasis, $n(\%)$ : } \\
\hline Single & $82(33.7)$ \\
\hline Multiple & $161(66.3)$ \\
\hline \multicolumn{2}{|l|}{ Metastatic site, $n(\%)$ : } \\
\hline Bone & $114(46.9)$ \\
\hline Liver & $104(42.8)$ \\
\hline Lung & $85(35.0)$ \\
\hline Brain & $37(15.2)$ \\
\hline Regional & $94(38.7)$ \\
\hline Chest wall & $81(33.3)$ \\
\hline Pleura & $26(10.7)$ \\
\hline Contralateral breast & $25(10.3)$ \\
\hline Skin and soft tissue & $38(15.6)$ \\
\hline Distant lymph node & $31(12.8)$ \\
\hline Other $^{\mathrm{a}}$ & $12(4.9)$ \\
\hline
\end{tabular}

ECOG - Eastern Cooperative Oncology Group, HER2 - human epidermal growth factor receptor 2, HR - hormone receptor. Sites of metastasis include single and multiple metastases. ${ }^{a}$ Other includes pericardium, spleen, adrenal, and ovary. *Including tubular carcinoma, mucinous carcinoma, and mixed carcinoma.

\section{Statistical analysis}

Statistical analyses were performed with SPSS 15.0 Statistics Software (SPSS Inc., an IBM company, Chicago, IL, USA). For categorical variables, data are presented as number (percent), and the $\chi^{2}$ test was used to test the relation between two binary variables. To determine the difference in age between HR positive and negative patients, independent two-sample $t$ test was applied and data are presented as mean \pm standard deviation (SD). Kaplan-Meier analysis with log-rank test was performed to create cumulative survival curves [23]. Univariate and multivariate Cox proportional hazards analyses of OS were performed to identify prognostic clinicopathologic factors for HER2+ metastatic breast cancer. Variables with a $p$ value $<0.05$ in univariate analysis were selected and evaluated by multivariate analysis. All statistical assessments were two-sided, and statistical significance was set at $p<0.05$.

\section{Results}

Clinical characteristics of the 243 patients with HER2+ metastatic breast cancer are summarized in Table I. The mean age at metastasis was 46.0 \pm 10.1 years (range: $26-76$ years), with more patients being premenopausal $(68.7 \%)$ than postmenopausal (31.3\%). The most common histologic type was invasive ductal carcinoma (91.4\%), followed by other $(7.4 \%)$ and invasive lobular carcinoma (1.2\%). For HR expression, 138 (56.8\%) patients were HR positive and 105 (43.2\%) patients were HR negative. After recurrence or metastasis, $214(88.1 \%)$ patients received chemotherapy, $87(35.8 \%)$ patients received endocrine therapy, 85 (35.8\%) patients received anti-HER2 treatment, and 63 (25.9\%) patients received surgical intervention. Most of the patients $(66.3 \%)$ had multiple metastases. Bone was the most common site for metastasis (46.9\%), followed by liver (42.8\%), regional (38.7\%), lung (35.0\%), and chest wall (33.3\%). The majority of patients $(92.2 \%)$ had an ECOG performance status score $\leq 1$.

During the follow-up period, 205 patients died, with a median OS of 27 months ( $95 \% \mathrm{Cl}: 23.5$, 30.5 months), and the 1-, 3-, and 5-year survival rates were $84.4 \%, 38.6 \%$, and $18.1 \%$, respectively (Figure $1 \mathrm{~A}$ ). The median OS of $\mathrm{HR}+$ patients was significantly higher than that of HR- patients (46.0 months vs. 21.0 months, $p<0.001$, Figure $1 \mathrm{~B}$ ). In addition, the metastatic patients who received surgery had a significantly higher median OS than patients without surgery (38 months vs. 17 months, $p<0.001$, Figure $1 \mathrm{C}$ ). In 85 patients who received anti-HER2 therapy, there was no significant difference in OS between $\mathrm{HR}+$ and $\mathrm{HR}-$ subgroups ( $p=0.794$, Figure $1 \mathrm{D})$. 
A

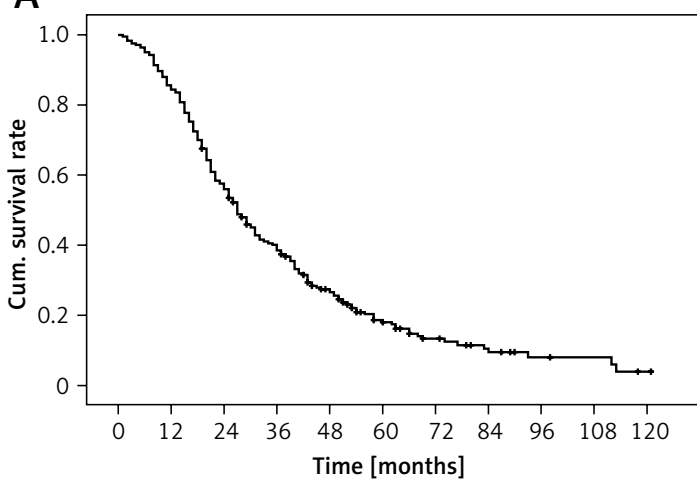

C

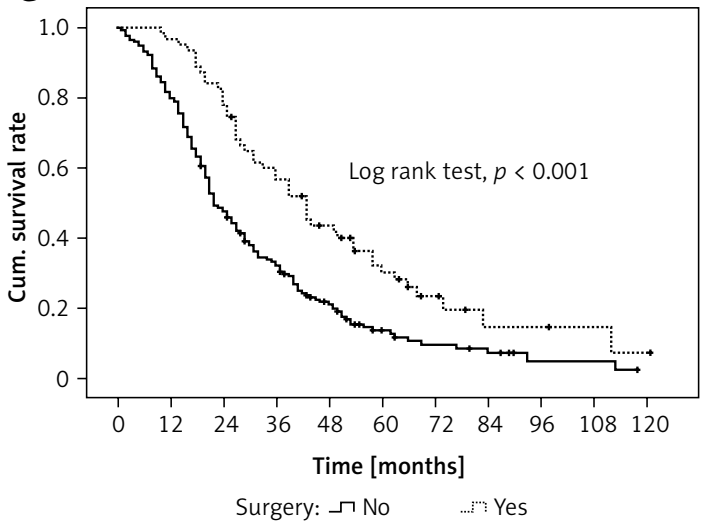

B

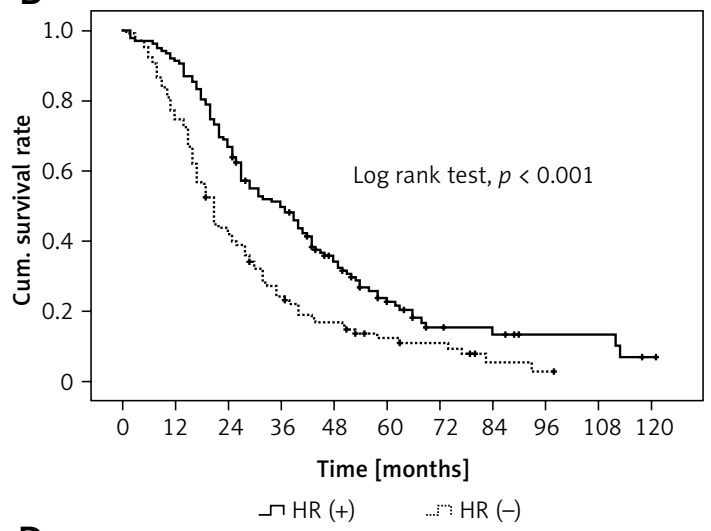

D

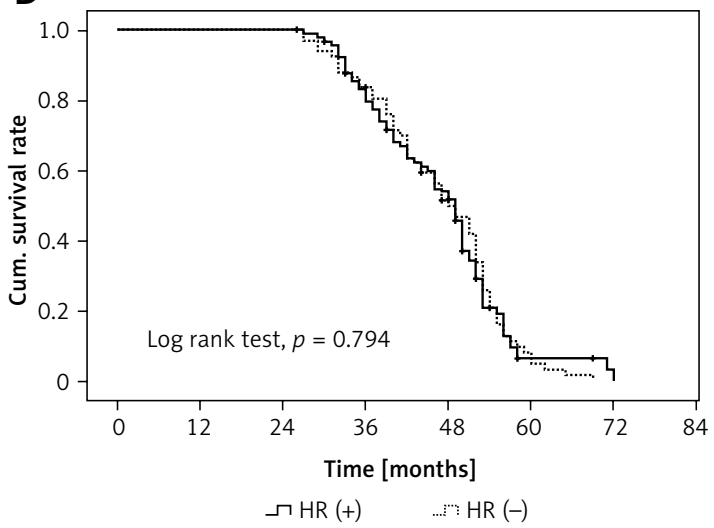

Figure 1. Cumulative survival curves. A - Overall survival. B - Overall survival in hormone receptor-positive (HR+) and negative (HR-) patients. C - Overall survival in patients with surgery and without surgery. D - Overall survival in $\mathrm{HR}+$ and $\mathrm{HR}$ - patients receiving anti-HER2 therapy

Next, potential prognostic factors for 243 patients with HER2-positive metastatic breast cancer were analyzed by univariate and multivariate Cox proportional hazards regression (Table II). Univariate Cox proportional hazards regression analysis identified the following prognostic factors: HR status, surgery, endocrine therapy, anti-HER2 therapy, ECOG performance status score, single versus multiple metastases, and brain metastasis $(p<0.05)$. These significant factors were selected for further multivariate Cox proportional hazards analysis. We found surgery (hazard ratio $=0.60$ $(0.43,0.83), p=0.002)$, endocrine therapy (hazard ratio $=0.53(0.37,0.74), p<0.01)$, and anti-HER2 therapy (hazard ratio $=0.63(0.47,0.85), p=0.003)$ as favorable independent prognostic factors, and ECOG performance status score $>1$ (hazard ratio $=3.62(2.13,6.16), p<0.001)$ and metastasis to brain (hazard ratio $=1.76(1.20,2.58), p=0.004)$ as unfavorable independent prognostic factors for patients with HER2-positive metastatic breast cancer.

\section{Discussion}

The aim of the present study was to identify factors that might affect prognosis for patients with HER2-positive metastatic breast cancer. The results of this retrospective study showed that surgical intervention, endocrine therapy, and antiHER2 therapy were favorable independent prognostic factors, whereas ECOG performance status $>1$ and metastasis to the brain were unfavorable independent prognostic factors. In the present study, all patients were HER2 positive and had advanced breast cancer, which generally has a poor prognosis. There are numerous factors that might affect prognosis in patients with HER2 overexpression, but the role of HR status in predicting prognosis is still unclear. In a study by Kaufman et al. [24], targeted therapy in combination with endocrine therapy was performed in patients who were positive for ER and had HER2 overexpression. Their findings revealed a superior outcome to that after endocrine therapy alone (progression-free survival: 4.8 vs. 2.4 months). However, a recent study by Dawood et al. [25] showed that among metastatic breast cancer patients who were HER2+, more HR- patients benefited from trastuzumab treatment compared to $\mathrm{HR}+$ patients. Our finding of greater OS for patients with $\mathrm{HR}+$ status is consistent with several recent reports of various patient subsets [7-10, 25]. Although HR+ status was favorable in comparison to HR- status according to univariate analysis, there was no signif- 
Table II. Cox proportional hazards regression analysis of potential prognostic factors for patients with HER2-positive metastatic breast cancer

\begin{tabular}{|c|c|c|c|c|}
\hline \multirow[t]{2}{*}{ Variable } & \multicolumn{2}{|c|}{ Univariate } & \multicolumn{2}{|c|}{ Multivariate } \\
\hline & Hazard ratio $(95 \% \mathrm{Cl})$ & Value of $p$ & Hazard ratio $(95 \% \mathrm{Cl})$ & Value of $p$ \\
\hline Age at metastasis & $0.99(0.98,1.01)$ & 0.304 & & \\
\hline \multicolumn{5}{|l|}{ Histologic type: } \\
\hline Invasive ductal vs. other & $0.78(0.48,1.29)$ & 0.337 & & \\
\hline Invasive lobular vs. other & $0.99(0.29,3.41)$ & 0.992 & & \\
\hline \multicolumn{5}{|l|}{ HR status: } \\
\hline $\mathrm{HR}(+)$ vs. $\mathrm{HR}(-)$ & $0.57(0.43,0.75)$ & $<0.001^{\star}$ & $0.81(0.59,1.11)$ & 0.182 \\
\hline \multicolumn{5}{|l|}{ Chemotherapy: } \\
\hline Yes vs. no & $1.22(0.77,1.92)$ & 0.392 & & \\
\hline \multicolumn{5}{|l|}{ Radiotherapy: } \\
\hline Yes vs. no & $0.76(0.57,1.01)$ & 0.057 & & \\
\hline \multicolumn{5}{|l|}{ Surgery: } \\
\hline Yes vs. no & $0.53(0.38,0.74)$ & $<0.001^{*}$ & $0.60(0.43,0.83)$ & $0.002^{*}$ \\
\hline \multicolumn{5}{|l|}{ Endocrine therapy: } \\
\hline Yes vs. no & $0.38(0.28,0.52)$ & $<0.001^{*}$ & $0.53(0.37,0.74)$ & $<0.001^{\star}$ \\
\hline \multicolumn{5}{|l|}{ Anti-HER2 therapy: } \\
\hline Yes vs. no & $0.64(0.47,0.85)$ & $0.003^{*}$ & $0.63(0.47,0.85)$ & $0.003^{*}$ \\
\hline \multicolumn{5}{|c|}{ ECOG performance status score: } \\
\hline$>1$ vs. $\leq 1$ & $4.80(2.88,7.98)$ & $<0.001^{*}$ & $3.62(2.13,6.16)$ & $<0.001^{*}$ \\
\hline \multicolumn{5}{|l|}{ Metastasis: } \\
\hline Multiple vs. single & $1.36(1.02,1.83)$ & $0.038^{*}$ & $1.16(0.86,1.57)$ & 0.332 \\
\hline \multicolumn{5}{|l|}{ Brain metastasis: } \\
\hline Yes vs. no & $1.95(1.35,2.82)$ & $<0.001^{\star}$ & $1.76(1.20,2.58)$ & $0.004^{\star}$ \\
\hline
\end{tabular}

icant difference in multivariate Cox proportional hazards regression analysis. In addition, anti-HER2 therapy showed similar OS for HR+ and HR- patients. There are few studies assessing HR status and response to anti-HER2 therapy; however, the present results are consistent with published results $[8,26]$. Additional studies are necessary to determine whether HR status affects the response to anti-HER2 therapy. Interestingly, we found that surgery and endocrine therapy can improve prognosis for HER2-positive patients with advanced breast cancer.

It is not surprising to find that anti-HER2 therapy was favorable for HER2-positive patients with metastatic breast cancer in the present study. Though trastuzumab can significantly improve the OS of breast cancer patients with HER2 overexpression, the incidence of brain metastasis has been noted to be increased (28-40\%) in this pa- tient population [27]. The identification of brain metastasis is consistent with a previous study of brain metastases in Chinese patients with breast cancer, which indicated poor prognosis of those with HER2+ status [28]. A previous study has shown that the incidence of brain metastasis is approximately $10 \%$ to $15 \%$ in HER2+ patients [29]. Our results showed that the incidence of brain metastasis was as high as $15.23 \%$ and supported the observation that the presence of brain metastasis is an unfavorable prognostic factor in HER2+ patients.

The present study also showed that ECOG performance status $>1$ was an unfavorable prognostic factor, consistent with previous studies [30, 31]. However, multivariate analysis showed that late stage at initial diagnosis and the number of metastatic lesions were not significant prognostic factors. Whereas the stage of breast cancer at 
initial diagnosis affects the selection of adjuvant therapy, with the application of surgery in patients with early breast cancer and the use of postoperative adjuvant therapy, the stage of breast cancer now has a reduced influence on survival. With the exception of brain metastasis, metastatic site and the number of metastatic lesions failed to influence breast cancer prognosis. There is evidence that in patients with controlled liver metastasis, focal therapy can prolong survival time [32]. Progression with focal therapy significantly improves survival in patients with focal recurrence and in a portion of patients with distant metastasis. In the present study, although the incidence of liver metastasis was at a high level, few patients received focal therapy, which is not representative.

The present study is limited by the fact that it was retrospective in nature, with a relatively small sample size. In addition, not all patients could receive anti-HER2 therapy due to financial reasons, which could have affected the survival analysis. Nonetheless, the major findings were consistent with those of previous studies, indicating prognostic similarities between Chinese patients with HER2 + metastatic breast cancer and other patient subsets. The findings for this specific population with HER2+ advanced breast cancer add to the overall knowledge base concerning prognostic factors in breast cancer, and might help with the tailoring of individualized therapy regimens.

In conclusion, our present results indicate that for patients with HER2+ metastatic breast cancer in southern China, OS is related to biologic behaviors of breast cancer as well as the type of metastasis and the therapy regimen. In particular, surgical intervention, endocrine therapy, and anti-HER2 therapy were favorable independent prognostic factors, and ECOG performance status > 1 and metastasis to the brain were unfavorable independent prognostic factors. HR status was not an independent prognostic factor in these patients.

\section{Acknowledgments}

Tao Qin and Zhong-yu Yuan contributed equally to this study.

Funding sources: National natural science foundation of China (81272896).

\section{Conflict of interest}

The authors declare no conflict of interest.

\section{References}

1. GLOBOCAN 2008, International Agency for Research on Cancer. Available at http://globocan.iarc.fr/factsheets/ cancers/breast.asp\#INCIDENCE. Accessed May 6, 2013.

2. Slamon DJ, Clark GM, Wong SG, Levin WJ, Ullrich A, McGuire WL. Human breast cancer: correlation of relapse and survival with amplification of the her-2/neu oncogene. Science 1987; 235: 177-82.

3. Slamon DJ, Godolphin W, Jones LA, et al. Studies of the HER-2/neu proto-oncogene in human breast and ovarian cancer. Science 1989; 244: 707-12.

4. Ferretti G, Felici A, Papaldo P, Fabi A, Cognetti F. HER2/ neu role in breast cancer: from a prognostic foe to a predictive friend. Curr Opin Obstet Gynecol 2007; 19: 56-62.

5. Piotrowski G, Gawor R, Stasiak A, Gawor Z, Potemski P, Banach M. Cardiac complications associated with trastuzumab in the setting of adjuvant chemotherapy for breast cancer overexpressing human epidermal growth factor receptor type 2 - a prospective study. Arch Med Sci 2012; 8: 227-35.

6. Dunnwald LK, Rossing MA, Li C. Hormone receptor status, tumor characteristics, and prognosis: a prospective cohort of breast cancer patients. Breast Cancer Res 2007; 9: R6.

7. Carey LA, Perou CM, Livasy CA, et al. Race, breast cancer subtypes, and survival in the Carolina Breast Cancer Study. JAMA 2006; 295: 2492-502.

8. Untch M, Gelber RD, Jackisch C, et al. Estimating the magnitude of trastuzumab effects within patient subgroups in the HERA trial. Ann Oncol 2008; 19: 1090-6.

9. Park YH, Lee S, Cho EY, et al. Patterns of relapse and metastatic spread in HER2-overexpressing breast cancer according to estrogen receptor status. Cancer Chemother Pharmacol 2010; 66: 507-16.

10. Gómez HL, Castañeda CA, Vigil CE, et al. Prognostic effect of hormone receptor status in early HER2 positive breast cancer patients. Hematol Oncol Stem Cell Ther 2010; 3: 109-15.

11. Kołacińska A, Chałubińska J, Błasińska-Morawiec M, et al. Pathological complete response in younger and older breast cancer patients. Arch Med Sci 2012; 8: 310-5.

12. Kwong M, Mang OW, Wong CH. Breast cancer in Hong Kong, Southern China: the first population-based analysis of epidemiological characteristics, stage-specific, cancer-specific, and disease-free survival in breast cancer patients: 1997-2001. Ann Surg Oncol 2011; 18: 3072-8.

13. Xu YL, Sun Q, Shan GL, et al. A case-control study on risk factors of breast cancer in China. Arch Med Sci 2012; 8: 303-9.

14. Su Y, Zheng Y, Zheng W, et al. Distinct distribution and prognostic significance of molecular subtypes of breast cancer in Chinese women: a population-based cohort study. BMC Cancer 2011; 11: 292.

15. Wong FY, Yip CS, Chua ET. Implications of HER2 amplification in small, node-negative breast cancers: do Asians differ? World I Surg 2012; 36: 287-94.

16. Liu AN, Sun P, Liu JN, et al. Clinicopathologic characteristics and prognostic factors in patients with operable HER-2 overexpressing breast cancer. Asian Pacific J Cancer Prev 2012; 13: 1197-201.

17. Chen XS, Wu JY, Huang O, et al. Molecular subtype can predict the response and outcome of Chinese locally advanced breast cancer patients treated with preoperative therapy. Oncol Rep 2010; 23: 1213-20.

18. Tang LC, Yin WJ, Di GH, Shen ZZ, Shao ZM. Unfavorable clinicopathologic features and low response rate to systemic adjuvant therapy: results with regard to poor survival in young Chinese breast cancer patients. Breast Cancer Res Treat 2010; 122: 95-104.

19. Telli ML, Chang ET, Kurian AW, et al. Asian ethnicity and breast cancer subtypes: a study from the California Cancer Registry. Breast Cancer Res Treat 2011; 127: 471-8. 
20. Edge SB, Byrd DR, Compton CC (eds.). AJCC cancer staging manual. 7th ed. Springer, New York (NY) 2009.

21. Therasse P, Arbuck SG, Eisenhauer EA, et al. New guidelines to evaluate the response to treatment in solid tumors. J Natl Cancer Inst 2000; 92: 205-16.

22. Oken MM, Creech RH, Tormey DC, et al. Toxicity and response criteria of the Eastern Cooperative Oncology Group. Am J Clin Oncol 1982; 5: 649-55.

23. Kaplan EL, Meier P. Nonparametric estimation from incomplete observations. J Am Stat Assoc 1958; 53: 457-81.

24. Kaufman B, Mackey JR, Clemens MR. Trastuzumab plus anastrozole versus anastrozole alone for the treatment of postmenopausal women with human epidermal growth factor receptor 2-positive, hormone receptorpositive metastatic breast cancer: results from the randomized phase III tandem study. J Clin Oncol 2009; 27: 5529-37.

25. Dawood S, Broglio K, Buzdar AU, Hortobagyi GN, Giordano SH. Prognosis of women with metastatic breast cancer by HER2 status and trastuzumab treatment: an institutional-based review. J Clin Oncol 2010; 28: 92-8.

26. Romond EH, Perez EA, Bryant J, et al. Trastuzumab plus adjuvant chemotherapy for operable HER2-positive breast cancer. N Engl J Med 2005; 353: 1673-84.

27. Bendell JC, Domchek SM, Burstein HJ, et al. Central nervous system metastases in women who receive trastuzumab-based therapy for metastatic breast carcinoma. Cancer 2003; 97: 2972-7.

28. Bai B, Yuan ZY, Liu DG, Teng XY, Wang SS. Clinical features and survival analysis of different subtypes of patients with breast cancer brain metastases. Chin J Cancer 2010; 29: 413-9.

29. Dawood S, Broglio K, Esteva FJ, et al. Defining prognosis for women with breast cancer and CNS metastases by HER2 status. Ann Oncol 2008; 19: 1242-8.

30. Largillier R, Ferrero JM, Doyen J, et al. Prognostic factors in 1,038 women with metastatic breast cancer. Ann Oncol 2008; 19: 2012-9.

31. Chang J, Clark GM, Allred DC, Mohsin S, Chamness G, Elledge RM. Survival of patients with metastatic breast carcinoma: importance of prognostic markers of the primary tumor. Cancer 2003; 97: 545-53.

32. Lubrano J, Roman H, Tarrab S, Resch B, Marpeau L, Scotté M. Liver resection for breast cancer metastasis: does it improve survival? Surg Today 2008; 38: 293-9. 